Check for updates

Cite this: Phys. Chem. Chem. Phys.,

2021, 23, 5447

Received 5th January 2021,

Accepted 22nd February 2021

DOI: $10.1039 / \mathrm{d} 1 \mathrm{cp} 00041 \mathrm{a}$

rsc.li/pccp

\title{
On the population of triplet states of 2-seleno-thymine $\dagger$
}

\author{
Danillo Valverde, (D) a Sebastian Mai, (D) *bc \\ Adalberto Vasconcelos Sanches de Araújo, (D) d Sylvio Canuto, (D) a \\ Leticia González (D) ${ }^{c}$ and Antonio Carlos Borin (D)*d
}

\begin{abstract}
The population and depopulation mechanisms leading to the lowest-lying triplet states of 2-Se-Thymine were studied at the MS-CASPT2/cc-pVDZ level of theory. Several critical points on different potential energy hypersurfaces were optimized, including minima, conical intersections, and singlet-triplet crossings. The accessibility of all relevant regions on the potential energy hypersurfaces was investigated by means of minimum energy paths and linear interpolation in internal coordinates techniques. Our analysis indicates that, after the population of the bright $S_{2}$ state in the Franck-Condon region, the first photochemical event is a barrierless evolution towards one of its two minima. After that, three viable photophysical deactivation paths can take place. In one of them, the population in the $\mathrm{S}_{2}$ state is transferred to the $T_{2}$ state via intersystem crossing and subsequently to the $T_{1}$ state by internal conversion. Alternatively, the $S_{1}$ state could be accessed by internal conversion through two distinct conical intersections with $S_{2}$ state followed by singlet-triplet crossing with the $T_{2}$ state. The absence of a second minimum on the $T_{1}$ state and a small energy barrier on pathway along the potential energy surface towards the ground state from the lowest triplet state are attributed as potential reasons to explain why the lifetime of the triplet state of 2 -Se-Thymine might be reduced in comparison with its thio-analogue.
\end{abstract}

\section{Introduction}

In DNA and RNA, the genetic information ${ }^{1,2}$ is encoded by the sequence of the canonical nucleobases, which are purine and pyrimidine derivatives. These canonical nucleobases absorb in the ultra-violet (UV) region, meaning that they could in principle undergo harmful photochemical reactions. Fortunately, these canonical nucleobases have a native and very efficient selfprotection mechanism that brings them back to the ground state nonradiatively on an ultrafast timescale after photoexcitation., Here, conical intersections act as the photophysical decay funnels

\footnotetext{
${ }^{a}$ Institute of Physics, University of São Paulo, Rua do Matão 1371. 05508-090, São Paulo, SP, Brazil

${ }^{b}$ Photonics Institute, Vienna University of Technology, Gußhausstraße 27-29, 1040 Vienna, Austria

${ }^{c}$ Institute of Theoretical Chemistry, Faculty of Chemistry, University of Vienna, Währinger Straße 17, 1090 Vienna, Austria.E-mail: sebastian.mai@univie.ac.at

${ }^{d}$ Department of Fundamental Chemistry, Institute of Chemistry, University of São Paulo, Av. Prof. Lineu Prestes 748. 05508-000, São Paulo, SP, Brazil. E-mail:ancborin@iq.usp.br

$\dagger$ Electronic supplementary information (ESI) available: The following files are available free of charge: (i) comparison of vertical excitation energies calculations with different active spaces and atomic basis set; (ii) comparison of the groundstate geometry using two atomic basis set; (iii) Cartesian coordinates of the optimized structures. See DOI: 10.1039/d1cp00041a
}

that enable the efficient population transfer among different electronic states. ${ }^{5,6}$

Replacement of a carbonyl oxygen atom by a sulfur transforms a canonical nucleobase into a so-called thiobase. It has been shown that this strongly boosts intersystem crossing (ISC), leading to an increased triplet yield after excitation. ${ }^{7,8}$ Triplet states are often very reactive, which can be harmful but can also be taken advantage of in photodynamical therapy ${ }^{9}$ - a medicinal technique widely employed in the treatment of several diseases. ${ }^{10-14}$ Hence, thiobases have been extensively studied in the last years-both experimentally and theoretically ${ }^{15-20}$-with focus on their prospective suitability as photosensitizers for photodynamical therapy.

Recent experimental $^{21}$ and theoretical investigations ${ }^{22-26}$ have shown that selenium-substituted nucleobases (selenobases) could be even more effective photosensitizers. This is due to their near-unity triplet yields and their red-shifted into the visible absorption spectra (relative to the thiobases), thus increasing the possible light penetration into biological tissue. ${ }^{27}$ Accordingly, a comprehensive understanding of the population pathways after light irradiation is dearly needed, if selenobases are to be exploited in practical application as photosensitizers. Recently, some of us carried out a comprehensive analysis of the unusual photophysical properties of 2-Se-Uracil (2SeUra), 
showing that it has some potential as a photosensitizer. ${ }^{24}$ Here we aim at investigating the photodeactivation mechanisms of 2-Se-Thymine (2SeThy).

Uracil and thymine (5-methyl-uracil) differ only in the presence of a methyl group at the C5 position in thymine. It has been shown in detail that the presence of the methyl group (and other chemical modifications) on C5 position, significantly affects the excited-state decay due to subtle changes in the potential energy surfaces that in turn affect the nuclear motion, leading to slower deactivation and in general to a more complex relaxation mechanism for thymine as compared to uracil. ${ }^{6,28-31}$ This pushed us to investigate the effects of methylation on the triplet population mechanisms of 2-Se-Uracil. ${ }^{24}$

Some preliminary aspects of the 2SeThy ISC mechanisms were already investigated theoretically by Pirillo and coworkers, ${ }^{22}$ but it focused only on the Franck-Condon region. In this paper we carry out a comprehensive analysis of the global potential energy surfaces (PESs) of 2SeThy, mapping state minima, minimum energy paths and minimum energy crossing regions, allowing us to derive photophysical relaxation mechanisms. To this aim, we employ accurate multiconfigurational methods that include both static and dynamic electron correlation.

\section{Computational details}

Both the computation of vertical excitation energies (VEEs) and the optimization of critical points of the PESs were carried out using the multi-state complete active space perturbation theory of second order $^{32}$ (MS-CASPT2) method. The VEEs were calculated with the cc-pVDZ ${ }^{33}$ basis set and without relativistic effects, as well as with the relativistic ANO-RCC-VDZP ${ }^{34}$ basis set and considering relativistic effects following the DouglasKroll-Hess formalism. ${ }^{35-37}$ Since the results of both basis sets are very similar (see Tables $\mathrm{S} 1$ and $\mathrm{S} 2$ in $\mathrm{ESI} \dagger$ ), all subsequent steps were performed with the less expensive cc-pVDZ basis set. Two-electron integrals were computed with the Cholesky decomposition method ${ }^{38}$ to speed up the calculations.

Geometries were pre-optimized with the complete active space self-consistent field ${ }^{39}$ (CASSCF) method, employing an active space of 12 electrons in 9 orbitals (denoted as CAS(12,9)), comprising $8 \pi / \pi^{*}$ orbitals plus the lone pair of the selenium atom, as shown in Fig. 1 (green background). The final optimization of minima and crossing points (and associated energy calculations) was carried out at the MS-CASPT2 level of theory with the same active space. Additionally, VEEs were computed with a larger $\operatorname{CAS}(14,11)$ active space, by augmenting the $\operatorname{CAS}(12,9)$ active space with the $\sigma$ and $\sigma^{*}$ orbitals localized on the $\mathrm{C}=$ Se group, see Fig. 1 (blue background). Test calculations indicated that the $n_{\mathrm{O}}$ lone pair localized on the oxygen atom remains virtually doubly occupied in all cases and hence was excluded from the active space (further details in Table S3 in $\mathrm{ESI} \dagger)$.

All calculations were carried out with the OpenMolcas ${ }^{40}$ package. As OpenMolcas does not average density matrices

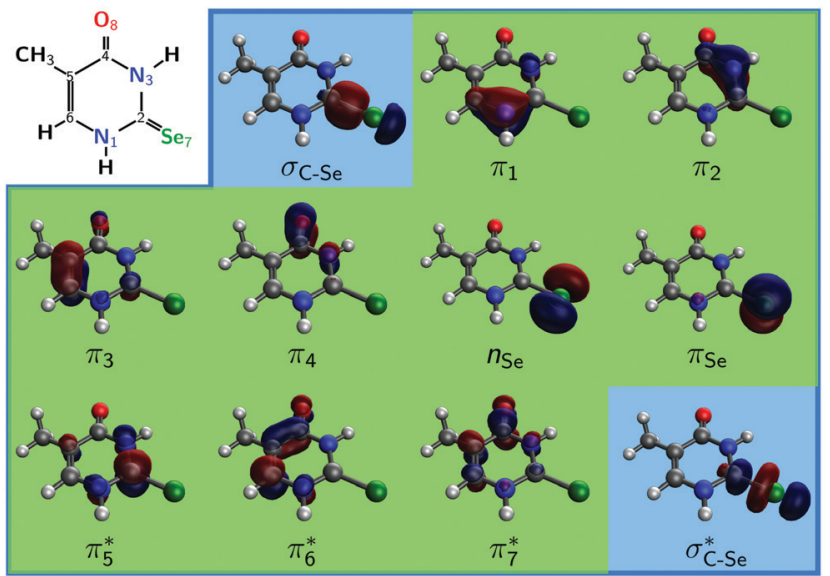

Fig. 1 Schematic structure of 2SeThy (with atom numbering) together to the active space orbitals employed in the calculations. The smaller CAS $(12,9)$ is shown with green background. The larger CAS $(14,11)$ additionally contains the two orbitals with blue background. Orbitals are shown for the ground state equilibrium geometry.

over states of different multiplicities, singlet and triplet states were computed separately, state-averaging over 4 singlet or 3 triplet states, respectively. An imaginary level-shift correction ${ }^{41}$ of 0.2 a.u. was used to prevent intruder states problems. Based on recommendations from literature, ${ }^{42}$ the IPEA shift ${ }^{43}$ was set to zero. Spin-orbit coupling (SOC) elements were computed through the AMFI (Atomic Mean Field Integrals) approach. ${ }^{44}$

Minimum energy crossing points (MECPs) were optimized with the restricted Lagrange multipliers technique. ${ }^{45}$ If the two states involved in a MECP structure have the same spin multiplicity, it is a conical intersection (CI) and if they belong to different spin symmetries (singlet and triplet, in our case), is a singlet-triplet crossing (STC). The initial relaxation pathway starting from the ground state minimum geometry was scanned with a minimum energy path (MEP) calculation (sphere of radius of 0.025 a.u.), carried out at the MS-CASPT2(12,9)/cc-pVDZ level of theory. Subsequent segments of the relaxation pathway were obtained by connecting the optimized structures with the linear interpolation in internal coordinates (LIIC) technique, computing the energies at each interpolated point at the same level of theory. One should note that the PES profiles obtained through LIIC scans only provide an upper limit for any energy barrier (as curved pathways with lower barriers could exist). However, the absence of any barrier from a LIIC scan segment can be taken as an unambiguously proof that no barrier is present.

\section{Results and discussion}

\subsection{Vertical excitation energies}

The computed VEEs and associated oscillator strengths of 2SeThy are shown in Table 1, together with other results available from the literature. All calculations predict that the lowest-lying excited state $\mathrm{S}_{1}$ has ${ }^{1} \mathrm{n}_{\mathrm{Se}} \pi_{5}^{*}$ character, i.e. it derives from a single excitation from the non-bonding orbital $\left(\mathrm{n}_{\mathrm{Se}}\right)$ 
Table 1 Vertical excitation energies $\Delta E_{\mathrm{VEE}}$ (eV, oscillator strengths in parenthesis) of 2SeThy obtained in gas phase at different levels of theory

\begin{tabular}{|c|c|c|c|c|}
\hline & Ref. 22 & Ref. 46 & This work & This work \\
\hline State & M06-2X & $\begin{array}{l}\text { MS-CASPT2 } \\
(14,10)\end{array}$ & $\begin{array}{l}\text { MS-CASPT2 } \\
(12,9)\end{array}$ & $\begin{array}{l}\text { MS-CASPT2 } \\
(14,11)\end{array}$ \\
\hline $\mathrm{S}_{1}\left({ }^{1} \mathrm{n}_{\mathrm{Se}} \pi_{5}^{*}\right)$ & 3.43 & $3.74(0.00)$ & $3.48(0.000)$ & $3.40(0.000)$ \\
\hline $\mathrm{S}_{2}\left({ }^{1} \pi_{\mathrm{Se}} \pi_{5}^{*} /{ }^{1} \pi_{\mathrm{Se}} \pi_{6}^{*}\right)$ & 3.97 & $4.48(0.50)$ & $3.96(0.549)$ & $3.91(0.265)$ \\
\hline $\mathrm{S}_{3}\left({ }^{1} \pi_{\mathrm{Se}} \pi_{6}^{*}\right)$ & - & $4.90(0.23)$ & $4.29(0.160)$ & $4.30(0.241)$ \\
\hline $\mathrm{T}_{1}\left({ }^{3} \pi_{\mathrm{Se}} \pi_{5}^{*} /{ }^{3} \pi_{\mathrm{Se}} \pi_{6}^{*}\right)$ & 2.91 & - & 2.97 & 2.85 \\
\hline $\mathrm{T}_{2}\left({ }^{3} \mathrm{n}_{\mathrm{Se}} \pi_{5}^{*}\right)$ & 2.98 & - & 3.44 & 3.29 \\
\hline
\end{tabular}

situated on the Se atom to the $\pi_{5}^{*}$ molecular orbital (Fig. 1). The low oscillator strength identifies it as a dark state. It is interesting to note that our results agree nicely with those reported by Pirillo $e$ e $a .^{22}$ at the M062X/6-31+G* level of theory, whereas those obtained by Manae and Hazra ${ }^{46}$ with MS-CASPT2 $(14,10) /$ cc-pVTZ (IPEA $=0.25$ a.u.) are overestimated by about $0.2 \mathrm{eV}$, probably due to the different IPEA shift and basis set employed.

The second excited state $\left(\mathrm{S}_{2}\right)$ is predicted at $3.91 \mathrm{eV}$ at our highest level of theory (MS-CASPT2 $(14,11)$ ) and is a bright state. Its character is a linear combination of two single excitations, ${ }^{1} \pi_{\mathrm{Se}} \pi_{5}^{*}(44 \%)$ and ${ }^{1} \pi_{\mathrm{Se}} \pi_{6}^{*}$ (45\%). As it is associated with the largest computed oscillator strength $(0.265)$, the $S_{2}$ is designated the initially excited state in our relaxation mechanism. The VEE of $S_{2}$ is in line with experimental results obtained in ethanol, ${ }^{47}$ which report an intense absorption band peaking at $3.95 \mathrm{eV}(314 \mathrm{~nm})$. Our results are also consistent with the observation that 2SeThy shows an absorption red-shifted by about $0.5 \mathrm{eV}$ relative to the corresponding thiobase 2-thio-thymine. ${ }^{48}$

The $\mathrm{S}_{3}$ state is calculated $4.30 \mathrm{eV}$ vertically above the ground state, is also bright, and can be described as being of ${ }^{1} \pi_{\mathrm{Se}} \pi_{6}^{*}$ character. While MS-CASPT2 $(14,11)$ predicts similar oscillator strength for the $S_{2}$ and $S_{3}$ (0.265 and 0.241), other levels of theory calculate a considerable smaller oscillator strength for $\mathrm{S}_{3}$. This can be attributed to the fact that both states derive from contributions of the ${ }^{1} \pi_{\mathrm{Se}} \pi_{6}^{*}$ and ${ }^{1} \pi_{\mathrm{Se}} \pi_{5}^{*}$ transitions, which show very strong mixing (and hence intensity exchange) that depends sensitively on the geometry and level of theory. The $\mathrm{S}_{3}$ state could be populated by excitation energies higher than that employed experimentally ${ }^{47}(3.95 \mathrm{eV})$, although this is not the focus of the present work. Nonetheless, even if $S_{3}$ is populated, it is likely that this state decays quickly to the $S_{2}$ state, due to the small energy gap between these states that makes the presence of a conical intersection probable. Hence, the most relevant photochemical events would be governed by the $S_{2}$ potential energy hypersuface. Henceforth we shall focus only on the bright $\mathrm{S}_{2}$ state to discuss the photophysical deactivation pathways of 2SeThy.

The lowest $\mathrm{T}_{1}$ and $\mathrm{T}_{2}$ triplet states are computed at $2.85 \mathrm{eV}$ and $3.29 \mathrm{eV}$, respectively. The $\mathrm{T}_{1}$ is dominated by a ${ }^{3} \pi_{\mathrm{Se}} \pi_{5}^{*}$ configuration, whereas the $\mathrm{T}_{2}$ state has ${ }^{3} \mathrm{n}_{\mathrm{Se}} \pi_{6}^{*}$ character. The energy of the $T_{1}$ is in reasonable agreement regardless the level of theory employed. However, the excitation energy of the $T_{2}$ state is lower at the M062X/6-31+G* level of theory ${ }^{22}$ than that predicted by our multiconfigurational calculations. We also note that the inclusion of the $\sigma$ and $\sigma^{*}$ orbitals in the active space affects the $T_{1}$ and $T_{2}$, stabilizing them by 0.12 and $0.15 \mathrm{eV}$, respectively.

\subsection{Excited states and crossing regions}

Fig. 2 shows the geometry of the calculated critical points of 2SeThy. Key geometrical parameters of minima and MECPs with adiabatic excitation energies (AEEs) are displayed in Table 2. Ring deformations were analyzed with Cremer-Pople parameters ${ }^{49}$ and the Boeyens classification scheme. ${ }^{50}$ The out-of-plane movement of the selenium atom was described by the SeC2N1N4 pyramidalization angle, defined as 90 degrees minus the angle between the $\mathrm{Se}-\mathrm{C}$ bond and the normal vector of $\mathrm{C} 2-\mathrm{N} 1-\mathrm{N} 4 .^{51}$

The 2SeThy ground state optimized geometry $\left(\left(\mathrm{S}_{0}\right)_{\min }\right.$, Fig. 2a) is planar, with only the $\mathrm{CH}_{3}$ hydrogen atoms out of the molecular plane. The $\mathrm{C}=\mathrm{Se}$ bond length is $1.82 \AA$, significantly larger than observed for the analogous $\mathrm{C}=\mathrm{O}$ (about $1.21 \AA$ ) or $\mathrm{C}=\mathrm{S}$ (about $1.56 \AA$ ) double bonds. ${ }^{52}$

The minimum of the first excited state, denoted as $\left(\mathrm{S}_{1}\right)_{\min }$, is placed adiabatically $2.89 \mathrm{eV}$ above the ground state minimum. Its ring remains planar as in the ground state, but the selenium atom leaves the molecular plane by 40 degrees (Fig. 2b).

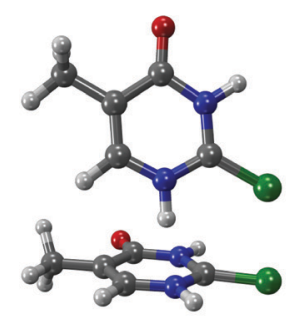

(a) Planar $\left(S_{0}\right)_{\min }$

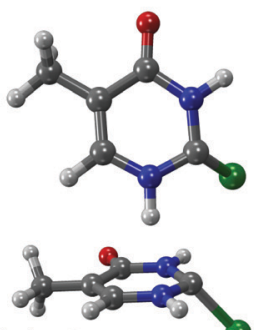

(b) $C_{2}$-pyramid

$\begin{array}{ll}\left(S_{1}\right)_{\min } & \left(S_{2}^{\text {pyr }}\right)_{\min } \\ \left(T_{1}\right)_{\min } & \left(T_{1} / T_{2}\right)_{\mathrm{Cl}} \\ \left(T_{2}\right)_{\min } & \left(S_{2}^{\text {prr }} / S_{1}\right)_{\mathrm{Cl}} \\ \left(S_{1} / T_{2}\right)_{\mathrm{STC}} & \left(S_{0} / S_{1}\right)_{\mathrm{Cl}}\end{array}$

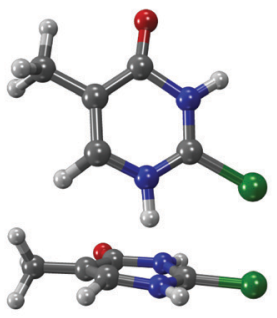

(c) Boat

$\left(S_{2}^{\text {boat }}\right)_{\min }$
$\left(S_{2}^{\text {boat }} / S_{1}\right)_{\mathrm{Cl}}$

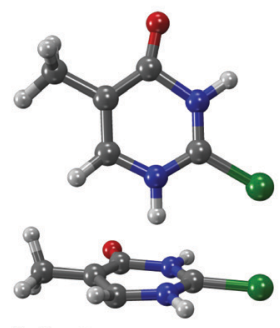

(d) $C_{6}$-pyramid $\left(S_{2} / T_{2}\right)_{\mathrm{STC}}$

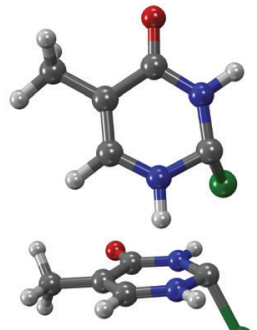

(e) $C_{2}$-vertical $\left(T_{1} / S_{0}\right)_{\mathrm{STC}}$

Fig. 2 Geometries of 2SeThy critical points in gas phase at the MS-CASPT2(12,9)/cc-pVDZ level of theory. 
Table 2 Adiabatic excitation energies ( $\Delta E$ in $\mathrm{eV}$ ) relative to the ground state minimum, bond distances $(\AA)$, pyramidalization angles (degree), Cremer-Pople parameters ( $Q$ in $\AA, \varphi$ and $\theta$ in degrees), and Boeyens conformation $^{50}$ for $2 \mathrm{SeThy}$ critical points in gas phase at the MSCASPT2(12,9)/cc-pVDZ level of theory

\begin{tabular}{|c|c|c|c|c|c|c|}
\hline \multirow[b]{2}{*}{ Parameters } & \multicolumn{6}{|c|}{ Minima } \\
\hline & $\left(\mathrm{S}_{0}\right)_{\min }$ & $\left(\mathrm{S}_{1}\right)_{\min }$ & $\left(\mathrm{S}_{2}^{\text {boat }}\right)_{\min }$ & $\left(\mathrm{S}_{2}^{\mathrm{pyr}}\right)_{\min }$ & $\left(\mathrm{T}_{1}\right)_{\min }$ & $\left(\mathrm{T}_{2}\right)_{\min }$ \\
\hline$\Delta E(\mathrm{eV})$ & 0.00 & 2.89 & 3.37 & 3.16 & 2.53 & 2.67 \\
\hline$r\left(\mathrm{~N}_{1} \mathrm{C}_{2}\right)$ & 1.374 & 1.394 & 1.330 & 1.372 & 1.395 & 1.383 \\
\hline$r\left(\mathrm{C}_{2} \mathrm{~N}_{3}\right)$ & 1.371 & 1.400 & 1.344 & 1.374 & 1.403 & 1.385 \\
\hline$r\left(\mathrm{~N}_{3} \mathrm{C}_{4}\right)$ & 1.415 & 1.402 & 1.496 & 1.410 & 1.398 & 1.406 \\
\hline$r\left(\mathrm{C}_{4} \mathrm{C}_{5}\right)$ & 1.465 & 1.471 & 1.420 & 1.469 & 1.472 & 1.471 \\
\hline$r\left(\mathrm{C}_{5} \mathrm{C}_{6}\right)$ & 1.368 & 1.369 & 1.433 & 1.369 & 1.372 & 1.366 \\
\hline$r\left(\mathrm{~N}_{1} \mathrm{C}_{6}\right)$ & 1.383 & 1.376 & 1.423 & 1.379 & 1.369 & 1.380 \\
\hline$r\left(\mathrm{C}_{2} \mathrm{Se}\right)$ & 1.823 & 1.987 & 1.920 & 2.120 & 1.988 & 2.074 \\
\hline$r\left(\mathrm{C}_{5} \mathrm{C}_{7}\right)$ & 1.502 & 1.502 & 1.504 & 1.500 & 1.501 & 1.501 \\
\hline$r\left(\mathrm{C}_{5} \mathrm{O}_{8}\right)$ & 1.225 & 1.228 & 1.239 & 1.225 & 1.229 & 1.226 \\
\hline$p\left(\mathrm{SeC}_{2} \mathrm{~N}_{1} \mathrm{~N}_{3}\right)$ & 0.1 & 40.0 & 2.1 & 48.9 & 44.2 & 42.1 \\
\hline$p\left(\mathrm{HC}_{6} \mathrm{~N}_{1} \mathrm{C}_{5}\right)$ & 0.2 & 1.8 & -7.4 & -0.2 & 0.2 & 1.5 \\
\hline$Q$ & 0.00 & 0.07 & 0.12 & 0.04 & 0.05 & 0.09 \\
\hline$\varphi$ & - & 72.5 & 115.3 & 128.9 & 70.2 & 135.3 \\
\hline$\theta$ & - & 73.8 & 99.9 & 65.0 & 64.7 & 57.9 \\
\hline Boeyens & - & ${ }^{3} \mathrm{~S}_{2}$ & $B_{3,6}$ & $E_{2}$ & $E_{2}$ & $E_{2}$ \\
\hline
\end{tabular}

\begin{tabular}{lcclllll}
\hline & \multicolumn{7}{c}{ Minimum energy crossing points } \\
\cline { 2 - 9 } & $\left(\mathrm{S}_{2}^{\text {boat }}\right.$ & $\left(\mathrm{S}_{2}^{\text {pyr }}\right.$ & $\left(\mathrm{S}_{0} /\right.$ & $\left(\mathrm{S}_{2} /\right.$ & $\left(\mathrm{S}_{1} /\right.$ & $\left(\mathrm{T}_{1} /\right.$ & $\left(\mathrm{T}_{1} /\right.$ \\
& $\left./ \mathrm{S}_{1}\right)_{\mathrm{CI}}$ & $\left(\mathrm{S}_{1}\right)_{\mathrm{CI}}$ & $\left.\mathrm{S}_{1}\right)_{\mathrm{CI}}$ & $\left.\mathrm{T}_{2}\right)_{\mathrm{STC}}$ & $\left.\mathrm{T}_{2}\right)_{\mathrm{STC}}$ & $\left.\mathrm{T}_{2}\right)_{\mathrm{CI}}$ & $\left.\mathrm{S}_{0}\right)_{\mathrm{STC}}$ \\
\hline$\Delta E(\mathrm{eV})$ & 3.47 & 3.23 & 3.53 & 3.44 & 2.92 & 2.77 & 2.73 \\
$r\left(\mathrm{~N}_{1} \mathrm{C}_{2}\right)$ & 1.308 & 1.366 & 1.436 & 1.331 & 1.403 & 1.390 & 1.441 \\
$r\left(\mathrm{C}_{2} \mathrm{~N}_{3}\right)$ & 1.306 & 1.367 & 1.414 & 1.326 & 1.397 & 1.389 & 1.413 \\
$r\left(\mathrm{~N}_{3} \mathrm{C}_{4}\right)$ & 1.467 & 1.413 & 1.396 & 1.563 & 1.398 & 1.403 & 1.388 \\
$r\left(\mathrm{C}_{4} \mathrm{C}_{5}\right)$ & 1.398 & 1.470 & 1.475 & 1.397 & 1.477 & 1.476 & 1.486 \\
$r\left(\mathrm{C}_{5} \mathrm{C}_{6}\right)$ & 1.425 & 1.370 & 1.363 & 1.441 & 1.367 & 1.364 & 1.355 \\
$r\left(\mathrm{~N}_{1} \mathrm{C}_{6}\right)$ & 1.434 & 1.379 & 1.370 & 1.421 & 1.381 & 1.391 & 1.372 \\
$r\left(\mathrm{C}_{2} \mathrm{Se}\right)$ & 1.920 & 2.157 & 2.299 & 1.922 & 1.965 & 2.020 & 2.043 \\
$r\left(\mathrm{C}_{5} \mathrm{C}_{7}\right)$ & 1.504 & 1.507 & 1.507 & 1.510 & 1.501 & 1.501 & 1.502 \\
$r\left(\mathrm{C}_{5} \mathrm{O}_{8}\right)$ & 1.223 & 1.224 & 1.229 & 1.229 & 1.227 & 1.226 & 1.228 \\
$p\left(\mathrm{SeC}_{2} \mathrm{~N}_{1} \mathrm{~N}_{3}\right)$ & 0.5 & 32.3 & 35.9 & 2.5 & 42.0 & 38.9 & 57.0 \\
$p\left(\mathrm{HC}_{6} \mathrm{~N}_{1} \mathrm{C}_{5}\right)$ & 10.7 & -0.3 & -3.9 & 9.3 & 1.7 & 2.5 & 0.9 \\
$Q$ & 0.06 & 0.05 & 0.14 & 0.08 & 0.10 & 0.28 & 0.07 \\
$\varphi$ & 78.4 & 126.8 & 116.1 & 64.5 & 70.7 & 32.3 & 52.5 \\
$\theta$ & 55.0 & 64.8 & 134.8 & 56.7 & 63.5 & 75.2 & 66.2 \\
Boeyens & ${ }^{5} H_{6}$ & $E_{2}$ & $E_{2}$ & $E_{6}$ & $E_{2}$ & ${ }^{1} \mathrm{~S}_{2}$ & $E_{2}$ \\
& & & & & & &
\end{tabular}

Also noticeable is the stretching of the $\mathrm{C}=$ Se bond by $0.16 \AA$ with respect to the equilibrium geometry.

At MS-CASPT2(12,9)/cc-pVDZ level, the $\mathrm{S}_{2}$ excited state PES exhibits two minima, as it was also observed for $2 \mathrm{TUra}^{53}$ and 2SeUra. ${ }^{24}$ The first minimum, with an AEE of $3.37 \mathrm{eV}$, has a boat conformation $\left(B_{3,6}\right)$; thus, we labeled it $\left(S_{2}^{\text {boat }}\right)_{\min }$ (Fig. 2c). This geometry shows a slight pyramidalization of the hydrogen atom on the 6-position (about 7 degrees) and a moderate increase on the $\mathrm{C}=\mathrm{Se}$ bond (about $0.1 \AA$ ) with respect to the ground state minimum. The second minimum is located at a lower energy $(3.16 \mathrm{eV})$ and exhibits strong out-of-plane displacement of the Se atom, as given by the pyramidalization angle of $\mathrm{C}_{2}$ of about 50 degrees. Thus, we label this minimum as $\left(S_{2}^{\mathrm{pyr}}\right)_{\min }$ (Fig. 2b).

The $\mathrm{T}_{1}$ optimized geometry $\left(\mathrm{T}_{1}\right)_{\mathrm{min}}$ is also characterized by a large pyramidalization (44.2 degrees) on the selenium atom (Fig. 2b). We failed to locate a second minimum on the $\mathrm{T}_{1}$ PES-as was reported for 2-thiothymine ${ }^{54,55}$ and other thiobases ${ }^{56}$-but consistent with the findings for 2 SeUra. ${ }^{24}$ This is of high relevance, as the presence and energy of the $T_{1}$ minima is decisive for the $T_{1}$ life time and the decay back to the ground state. Indeed, the presence of only one $T_{1}$ minimum in 2SeThy and 2SeUra could be a reason for their strongly decreased triplet life time as compared to their thio-analogues. ${ }^{21}$

We also optimized the MECPs involving the $S_{1}$ and $S_{2}$ states, obtaining two structures. For one of them, denoted $\left(\mathrm{S}_{2}^{\mathrm{pyr}} / \mathrm{S}_{1}\right)_{\mathrm{CI}}$ (Fig. 2b), we noted a large pyramidalization on the selenium atom, but not so pronounced as in the $\left(\mathrm{S}_{1}\right)_{\min }$ and $\left(\mathrm{S}_{2}^{\mathrm{pyr}}\right)_{\min }$ structures. The second structure, $\left(\mathrm{S}_{2}^{\text {boat }} / \mathrm{S}_{1}\right)_{\mathrm{CI}}$, is characterized by a boat conformation, resembling the $\left(\mathrm{S}_{2}^{\text {boat }}\right)_{\min }$ optimized minimum (Fig. 2c). The latter crossing is a bit different than that found for $2 \mathrm{SeUra},{ }^{24}$ because the bond distances in the ring are smaller for 2SeThy (by about $0.02 \AA$ ) as well as the pyramidalization angle (10 degrees versus 25 degrees). In addition, the optimized crossing structure between the $S_{0}$ and $S_{1}$ states $\left(\left(\mathrm{S}_{0} / \mathrm{S}_{1}\right)_{\mathrm{CI}}\right)$ (Fig. $\left.2 \mathrm{~b}\right)$ exhibits the largest $\mathrm{C}=$ Se bond among all optimized structures, with a pyramidalization on the selenium atom of $36^{\circ}$.

We have also located three STC structures. The first one involves the $S_{2}$ and $T_{2}$ states. This $\left(S_{2}^{\mathrm{pyr}} / \mathrm{T}_{2}\right)_{\mathrm{STC}}$ structure is slightly non-planar, which an envelope $\left(E_{6}\right)$ deformation and minor pyramidalization of the $\mathrm{C} 2$ (2.5 degrees) and $\mathrm{C} 6$ (9.3 degrees) atoms (Fig. 2d). The second STC $\left(\mathrm{S}_{1} / \mathrm{T}_{2}\right)_{\mathrm{STC}}$ involves the $S_{1}$ and $T_{2}$ states, with an envelope deformation $\left(E_{2}\right)$, out-of-plane position of the Se atom (pyramidalization angle of 42 degrees), and a C-Se bond length of $1.922 \AA$ (Fig. 2b). Thus, its geometry is very similar to $\left(\mathrm{S}_{1}\right)_{\mathrm{min}}$. The third STC structure involves the $T_{1}$ and $S_{0}$ states, thus is denoted the $\left(\mathrm{T}_{1} / \mathrm{S}_{0}\right)_{\mathrm{STC}}$, and features the largest pyramidalization on the selenium atom among all the optimized structures (Fig. 2e).

For the three STCs, we computed effective spin-orbit couplings (SOCs) as $\sqrt{1 / 3}$ times the norm of the vector containing all spinorbit matrix elements involving the multiplet components of the two involved states (see the ESI, $\uparrow$ for the corresponding equation). We note that this definition differs by the $\sqrt{1 / 3}$ factor from our preceding paper on $2 \mathrm{SeUra} .{ }^{24}$ At the $\left(\mathrm{S}_{2}^{\text {pyr }} / \mathrm{T}_{2}\right)_{\text {STC }}$ geometry, we obtained a SOC of about $477 \mathrm{~cm}^{-1}$ between $\mathrm{S}_{2}$ and $\mathrm{T}_{2}$. Likewise, for the $\left(\mathrm{S}_{1} / \mathrm{T}_{2}\right)_{\mathrm{STC}}$ STC a SOC of $239 \mathrm{~cm}^{-1}$ was obtained. Both values are in qualitative agreement with the Franck-Condon results reported by Pirillo et al. ${ }^{22}$ (B3LYP/6-31G*) as $433 \mathrm{~cm}^{-1}$ (the paper lists it as $750 \mathrm{~cm}^{-1}$, but this does not include the $\sqrt{1 / 3}$ factor we use). At the $\left(\mathrm{S}_{0} / \mathrm{T}_{1}\right)_{\text {STC }}$ geometry the SOC is computed to be about $450 \mathrm{~cm}^{-1}$ (Fig. 3).

\subsection{Mapping the potential energy surface}

In order to obtain plausible deactivation pathways, the optimized minima and MECPs were connected either by MEPs or LIICs. Fig. 3 shows all the discussed deactivation pathways. We note here that in all LIIC scans the $S_{3}$ state stayed always above $4.0 \mathrm{eV}$ relative to the $S_{0}$ minimum, and thus was not included in Fig. 3 .

PATH I begins with a barrierless MEP from the FranckCondon region along the $S_{2}$ PES to the $\left(S_{2}^{\text {boat }}\right)_{\min }$ structure at 


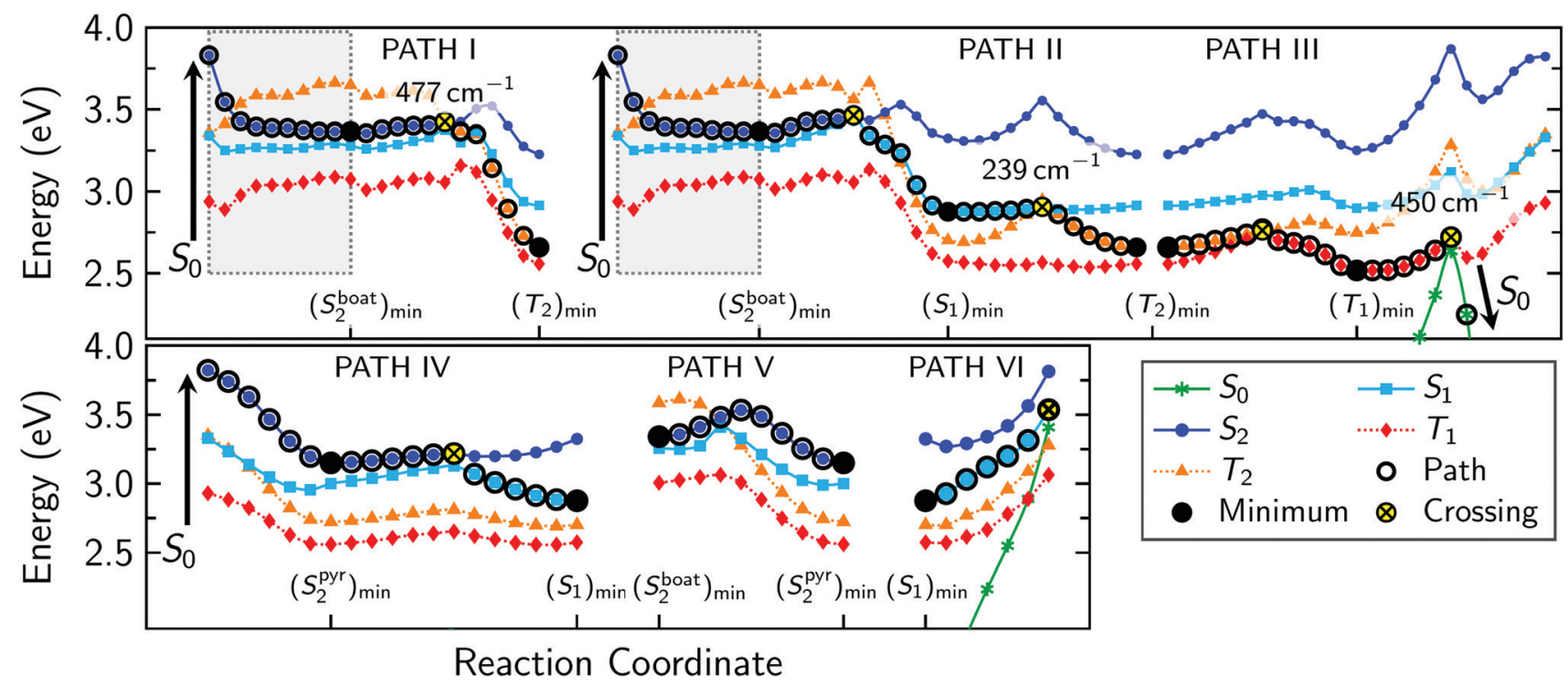

Fig. 3 Photophysical deactivation pathways of 2SeThy. At each structure obtained by MEP (gray background) and LIIC approaches, energies were computed at the MS-CASPT2(14,11)/cc-pVDZ level. Spin-orbit couplings (in $\mathrm{cm}^{-1}$ ) are reported for the crossing seam involving singlet and triplet states. Solid circles indicate the expected evolution of the system after light irradiation.

$3.37 \mathrm{eV}$. The path proceeds by linear interpolation to the $\left(\mathrm{S}_{2} / \mathrm{T}_{2}\right)_{\mathrm{STC}}$ crossing point, $0.07 \mathrm{eV}$ above the $\left(\mathrm{S}_{2}^{\text {boat }}\right)_{\min }$ structure. At the crossing point, the system might undergo ISC to the $\mathrm{T}_{2}$ state and subsequently relaxes to the $\left(\mathrm{T}_{2}\right)_{\min }$ that is $0.77 \mathrm{eV}$ below the crossing.

PATH II also starts with the MEP from the Franck-Condon region to $\left(\mathrm{S}_{2}^{\text {boat }}\right)_{\min }$, from where the system can access the $\left(S_{2}^{\text {boat }} / S_{1}\right)_{\mathrm{CI}}$ conical intersection that is located $0.10 \mathrm{eV}$ above the $S_{2}$ minimum. After internal conversion to the $S_{1}$ state, the system relaxes to the $\left(\mathrm{S}_{1}\right)_{\mathrm{min}}$, from where ISC to the $\mathrm{T}_{2}$ is easily possible at the $\left(\mathrm{S}_{1} / \mathrm{T}_{2}\right)_{\mathrm{STC}}$, which is only $0.03 \mathrm{eV}$ above the $\mathrm{S}_{1}$ minimum. After ISC, the system will first continue to the $\left(\mathrm{T}_{2}\right)_{\min }$. A direct ISC channel from $\mathrm{S}_{1}$ to $\mathrm{T}_{1}$-as hypothesized by Pirillo $e t$ al. ${ }^{22}$ based on the large SOC between these states at the Franck-Condon point-is very unlikely based on our computed relaxation pathways and intermediates.

It is interesting to note that an early $\left(\mathrm{S}_{2} / \mathrm{T}_{2}\right)_{\mathrm{STC}}$ can be observed in Paths I and II (Fig. 3). However, as this crossing is located on the steepest region of the path, there is not enough time for an efficient population transfer to take place. On the other hand, the early $S_{2} / T_{2}$ crossing is likely on the same seam as the later $S_{2} / T_{2}$ crossing in PATH I, which leads us to conclude that they would share the same decay path.

PATH III continues at the common endpoint of PATH I and PATH II, which is the $\left(\mathrm{T}_{2}\right)_{\mathrm{min}}$. Internal conversion to the $\mathrm{T}_{1}$ is possible at the $\left(\mathrm{T}_{2} / \mathrm{T}_{1}\right)_{\mathrm{CI}}$ conical intersection, $0.10 \mathrm{eV}$ above the $\mathrm{T}_{2}$ minimum. Subsequently, the molecule can access the $\left(\mathrm{T}_{1}\right)_{\min }$ region, at an energy of $2.53 \mathrm{eV}$ above the ground state minimum. As the $\left(\mathrm{T}_{1}\right)_{\min }$ is the lowest-energy excited-state minimum, it is likely decisive for the excited state life time of 2SeThy. However, the $\mathrm{T}_{1}$ can nonradiatively decay back to the ground state at the $\left(\mathrm{T}_{1} / \mathrm{S}_{0}\right)_{\text {STC }}$ crossing, placed $0.20 \mathrm{eV}$ above the $\left(\mathrm{T}_{1}\right)_{\min }$. This energy difference is in good agreement with the value reported for 2SeUra at the MS-CASPT2/cc-pVDZ level of theory. ${ }^{24}$
Although the MEP from the Franck-Condon region should be the most appropriate pathway to describe the initial relaxation, it is in principle possible that the momentum of the initial wave packet leads part of it along other pathways. For 2SeThy, PATH IV in Fig. 3 shows that from the Franck-Condon point also the second $S_{2}$ minimum, $\left(\left(S_{2}^{\text {boat }}\right)_{\min }\right)$, could be accessed in a barrierless fashion along a linearly interpolated pathway. Hence, a bifurcation of the initial wave packet into PATH I/II and PATH IV is rather likely. On the contrary, once either of the $S_{2}$ minima is reached, conversion to the other minimum is hampered by the energy barrier $(0.17 \mathrm{eV}$ above $\left.\left(\mathrm{S}_{2}^{\text {boat }}\right)_{\min }\right)$ shown in PATH V. In general, nonadiabatic molecular dynamics simulations would be necessary to judge which of these two directions is more relevant in the initial relaxation step. However, ultimately we do not expect a significantly different behaviour between PATH I/II and PATH IV, because also PATH IV eventually leads to $\left(\mathrm{S}_{1}\right)_{\mathrm{min}}$. This is possible due to the presence of the $\left(\mathrm{S}_{2} \mathrm{~S}_{2}^{\mathrm{pyr}} / \mathrm{S}_{1}\right)_{\mathrm{CI}}$ conical intersection, at an energy of $0.07 \mathrm{eV}$ above the $\left(\mathrm{S}_{2}{ }^{\mathrm{py}}\right)_{\min }$.

Finally, we analyzed the possibility of the system returning to the ground state via an internal conversion process, as shown in PATH-VI. As it can be seen, the LIIC path shows that this process is very unlikely because the $\left(\mathrm{S}_{1} / \mathrm{S}_{0}\right)_{\mathrm{CI}}$ is placed $0.42 \mathrm{eV}$ above the $\mathrm{S}_{1}$ state minimum structure. This finding is in agreement with previous theoretical works about thio- and seleno-nucleobases. ${ }^{18,57}$

In summary, after 2SeThy absorbs UV radiation, our calculations predict three possible deactivation mechanisms (I-III, II-III and IV-(II)-III in Fig. 3) that reach the lowest triplet state, see Fig. 4. Subsequently, a decay from the $T_{1}$ minimum to the ground state is possible via reverse ISC. This deactivation mechanism can be summarized as $\left(\mathrm{S}_{2}\right)_{\mathrm{FC}} \rightarrow \mathrm{S}_{2}^{\text {boat }} / \mathrm{S}_{2}^{\text {pyr }} \rightarrow \mathrm{S}_{1} \rightarrow \mathrm{T}_{2} \rightarrow \mathrm{T}_{1} \rightarrow \mathrm{S}_{0}$. Our calculations do not allow clarifying which of the two $\mathrm{S}_{2}$ minima constitutes the primary deactivation pathway, as this would require 


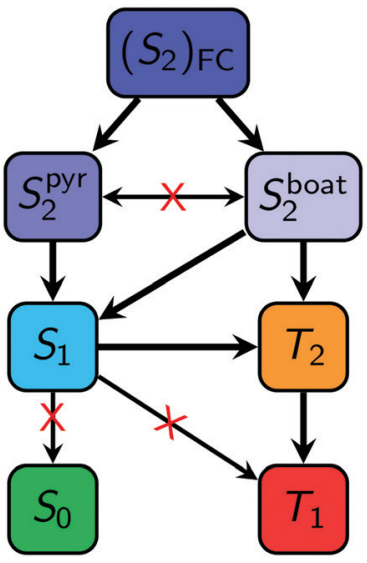

Fig. 4 Possible deactivation mechanisms of 2SeThy in gas phase from the $\mathrm{S}_{2}$, calculated at the MS-CASPT2(14,11)/cc-pVDZ//MS-CASPT2(12,9)/ cc-pVDZ level of theory.

explicit simulation of the nonadiabatic dynamics of the molecule. Lacking such simulations, we can only estimate the $\mathrm{S}_{2}$ minimum preference from the results published for $2 \mathrm{SeUra}^{24}$ at the $\mathrm{ADC}(2)$ level of theory, which suggest that the $\left(S_{2}^{\text {boat }}\right)_{\text {min }}$ region is only populated for a few femtoseconds before the trajectories turn towards the lower-energy $\left(\mathrm{S}_{2}^{\mathrm{pyr}}\right)_{\text {min }}$ region.

In comparison to $2 \mathrm{SeUra},{ }^{24}$ we can conclude that in this case methylation leads to minor effects on the excited-state PES of 2SeThy, as it only slightly reduces the energy barrier between the $\left(\mathrm{T}_{1}\right)_{\min }$ and $\left(\mathrm{T}_{1} / \mathrm{S}_{0}\right)_{\text {sTC }}$ structures. This energy barrier affects the life time of the triplet state, which we will discuss in more detail in the next section.

\subsection{Life time of the triplet state and dependence on the level of theory}

A final open issue concerns the life time of the 2SeThy triplet state in relation to that of 2 SeUra, both in gas phase and in solution. Here, we will scrutinize the dependence of the relevant energy barrier on the level of theory, in order to obtain a more comprehensive view on this issue. To this end, we have optimized the $\left(T_{1}\right)_{\min }$ and $\left(S_{0} / T_{1}\right)_{\text {STC }}$ structures for both molecules in water using different levels of theory, which comprises different active spaces, basis set, and imaginary level shift, including the effects of the environment by means of the Polarizable Continuum Model (PCM). ${ }^{58,59}$ These results are presented in Table 3.
In gas phase, the energy barrier for 2SeThy has a value smaller than that obtained for 2SeUra, although the difference is small. Therefore, the triplet states life time of 2SeThy in gas phase should be approximately equal or slightly shorter than the one of 2SeUra.

Our initial computation of the energy barrier between $\left(\mathrm{T}_{1}\right)_{\min }$ and $\left(\mathrm{S}_{0} / \mathrm{T}_{1}\right)_{\mathrm{STC}}$ regions in solution at the MSCASPT2(14,11)//MS-CASPT2(12,9)/cc-pVDZ level of theory yielded a value of $0.24 \mathrm{eV}$. As this value is significantly larger than the previously reported barrier of $0.06 \mathrm{eV}$ in $2 \mathrm{SeUra},{ }^{24}$ we recomputed the 2 SeUra barrier at our present level of theory and found $0.20 \mathrm{eV}$. In order to rule out a strong dependence of the barrier on the level of theory, we performed further optimizations for 2SeThy (see Table 3), which showed that the barrier depends slightly on the level of theory, varying between 0.14 and $0.24 \mathrm{eV}$. Importantly, the height of the barrier seems to be little affected by solvation in our new computations, as the different solution-phase results are generally consistent with the gas-phase results. Hence, we can deduce that the triplet states life time should be on the same order of magnitude in both environments. Similarly, we expect that 2SeThy and 2SeUra will have similar triplet life times in solution. Thereby, the conclusion of the previous work ${ }^{24}$ about the 2 SeUra should be revised.

Similar to the gas-phase results presented above, the optimized structures in water always lead to a single minimum in the $\mathrm{T}_{1}$ state. As discussed previously, ${ }^{24}$ this finding is at odds with that predicted for 2-thiouracil ${ }^{54}$ and 2-thiothymine. ${ }^{55}$ According to MS-CASPT2 calculations, both of these thiobases have two different minima on the $\mathrm{T}_{1}$ hypersurface, a boat-like one and a pyramidalized one. The presence of these two minima structures and the higher barriers to access the $T_{1} / S_{0}$ crossing point $\left(0.36 \mathrm{eV}^{54}\right.$ in 2-thiouracil and $0.30 \mathrm{eV}^{55}$ for 2-thiothymine) could enhance the trapping of the triplet population in the thiobases. Based on these findings, we conclude that the triplet state life time should be significantly shorter in 2SeThy than in 2-thiothymine.

\section{Conclusion}

In this contribution the photophysical deactivation pathways of 2SeThy was investigated at the MS-CASPT2/cc-pVDZ level of theory. Several excited-state minima and crossing points were found, establishing relaxation pathways that should lead to the

Table 3 Energy difference between the $\left(T_{1}\right)_{\min }$ and $\left(\mathrm{S}_{0} / \mathrm{T}_{1}\right)_{\text {sTc }}$ computed at different levels of theory for $2 \mathrm{SeThy}$ and $2 \mathrm{SeU}$ ra in gas phase and water

\begin{tabular}{|c|c|c|c|}
\hline & Level of theory & 2SeThy & 2SeUra \\
\hline
\end{tabular}


ultrafast population of the lowest triplet state after the absorption of light. Two mimina on the $S_{2}$ PES were found, an $\left(S_{2}^{\text {boat }}\right)$ structure and an $\left(S_{2}^{\text {pyr }}\right)$ structure. Even though our calculations cannot accurately predict which of those structures will be more relevant in the dynamics, both of them would allow for an efficient decay to the $S_{1}$ state or the triplet states. In summary, three feasible photophysical paths can be followed to populate the triplet state: (i) $\left(\mathrm{S}_{2}\right)_{\mathrm{FC}} \rightarrow \mathrm{S}_{2}^{\text {boat }} \rightarrow \mathrm{T}_{2} \rightarrow \mathrm{T}_{1}$; (ii) $\left(\mathrm{S}_{2}\right)_{\mathrm{FC}} \rightarrow \mathrm{S}_{2}^{\text {boat }} \rightarrow \mathrm{S}_{1} \rightarrow \mathrm{T}_{2} \rightarrow \mathrm{T}_{1}$; and (iii) $\left(\mathrm{S}_{2}\right)_{\mathrm{FC}} \rightarrow \mathrm{S}_{2}^{\text {pyr }} \rightarrow \mathrm{S}_{1} \rightarrow$ $\mathrm{T}_{2} \rightarrow \mathrm{T}_{1}$. Based on the presented energy profiles, we suggest that the latter is the preferential photochemical channel, although nonadiabatic dynamics simulations are required to confirm this prediction.

According to our results, the photochemical deactivation mechanisms of 2SeThy are very close to that determined for 2 SeUra, ${ }^{24}$ which implies that the effects due to methylation on the $\mathrm{C} 5$ position is rather small. Hence, we expect that both molecules will exhibit very similar triplet life times after excitation-both in gas phase and in solution-although 2SeThy might show slightly shorter life times due to its slightly lower activation barrier.

Compared to its thiobase analogue 2-thiothymine, in 2SeThy the absorption spectrum is red-shifted by about $0.5 \mathrm{eV}$, due to the weaker bound electrons in the $n_{\text {Se }}$ and $\pi_{\text {Se }}$ orbitals. The general relaxation mechanism after excitation should be very similar for 2SeThy, 2SeUra, 2-thiothymine, and 2-thiouracil. However, the two thiobases exhibit two rather deep minima ${ }^{53,54}$ on the $\mathrm{T}_{1}$ potential energy surface, that can more effectively trap population in the triplet state, compared to the single $\mathrm{T}_{1}$ minimum in the selenobases. Furthermore, the SOCs in the selenobases are approximately four times larger than in the thiobases. ${ }^{24,53,54}$ These two facts suggest that ISC from $T_{1}$ to $S_{0}$ should be significantly faster in the selenobases than in the thiobases. Such shorter triplet life times for selenobases were already reported for 6-selenoguanine, ${ }^{21}$ who also suggested that for this reason, selenobases might be preferentially employed as short-lived Type I photosensitizer that only affects its immediate vicinity.

\section{Conflicts of interest}

There are no conflicts to declare.

\section{Acknowledgements}

D. V. acknowledges financial support from the Fundação de Amparo à Pesquisa do Estado de São Paulo (FAPESP), under grants 2017/02612-4 and 2019/04413-4. S. C. thanks CAPES for the BioMol project 23038.004630/2014-35 and the National Institute of Science and Technology Complex Fluids (INCT-FCx) with the CNPq grant 141260/2017-3 and FAPESP grant 2014/ 50983-3. A. C. B. and A. V. S. A. thank the CNPq (Conselho Nacional de Desenvolvimento Científico e Tecnológico) for research fellowships (project numbers 302318/2017-8 and 153104/2015-5, respectively). A. C. B. thanks FAPESP for the research grant 2018/19454-5. Computations were performed on resources provided by HPC through Superintendência de Tecnologia da Informação da Universidade de São Paulo. The authors acknowledge the National Laboratory for Scientific Computing (LNCC/MCTI, Brazil) for providing HPC resources of the SDumont supercomputer (http://sdumont.lncc.br), which have contributed to the research results reported within this paper. S. M. and L. G. gratefully acknowledge the Vienna Scientific Cluster (VSC3) for allocation of computational resources and the University of Vienna for continuous support.

\section{References}

1 A. Rich, Q. Rev. Biophys., 2009, 42, 117-137.

2 A. Travers and G. Muskhelishvili, FEBS J., 2015, 282, 2279-2295.

3 C. E. Crespo-Hernández, B. Cohen, P. M. Hare and B. Kohler, Chem. Rev., 2004, 104, 1977-2020.

4 D. Tuna, A. L. Sobolewski and W. Domcke, J. Phys. Chem. A, 2014, 118, 122-127.

5 A. Giussani, J. Segarra-Martí, D. Roca-Sanjuán and M. Merchán, in Excitation of Nucleobases from a Computational Perspective I: Reaction Paths, ed. M. Barbatti, A. C. Borin and S. Ullrich, Springer International Publishing, Cham, 2015, pp. 57-97.

6 S. Mai, M. Richter, P. Marquetand and L. González, in Excitation of Nucleobases from a Computational Perspective II: Dynamics, ed. M. Barbatti, A. C. Borin and S. Ullrich, Springer International Publishing, Cham, 2015, pp. 99-153.

7 B. Ashwood, M. Pollum and C. E. Crespo-Hernández, Photochem. Photobiol., 2019, 95, 33-58.

8 R. Brem, F. Li and P. Karran, Nucleic Acids Res., 2009, 37, 1951-1961.

9 R. Darlenski and J. W. Fluhr, J. Biomed. Opt., 2012, 18, 061208. 10 L. P. Rosa and F. C. da Silva, J. Med. Microbiol., 2014, 3, 1.

11 M. Kim, H. Y. Jung and H. J. Park, Int. J. Mol. Sci., 2015, 16, 23259-23278.

12 A. Oniszczuk, K. A. Wojtunik-Kulesza, T. Oniszczuk and K. Kasprzak, Biomed. Pharmarcother., 2016, 83, 912-929.

13 S. B. Brown, E. A. Brown and I. Walker, Lancet Oncol., 2004, 8, 497-508.

14 S. Yano, S. Hirohara, M. Obata, Y. Hagiya, S. I. Ogura, A. Ikeda, H. Kataoka, M. Tanaka and T. Joh, J. Photochem. Photobiol., C, 2011, 12, 46-67.

15 H. Abrahamse and M. R. Hamblin, Biochem. J., 2016, 473, 347-364.

16 Y. Harada, C. Okabe, T. Kobayashi, T. Suzuki, T. Ichimura, N. Nishi and Y.-Z. Xu, J. Phys. Chem. Lett., 2010, 1, 480-484.

17 Y. Harada, T. Suzuki, T. Ichimura and Y.-Z. Xu, J. Phys. Chem. B, 2007, 111, 5518-5524.

18 S. Mai, M. Pollum, L. Martínez-Fernández, N. Dunn, P. Marquetand, I. Corral, C. E. Crespo-Hernández and L. González, Nat. Commun., 2016, 7, 13077.

19 A. Mohamadzade, S. M. Bai, M. Barbatti and S. Ullrich, Chem. Phys., 2018, 515, 572-579. 
20 H. Kuramochi, T. Kobayashi, T. Suzuki and T. Ichimura, J. Phys. Chem. B, 2010, 114, 8782-8789.

21 K. M. Farrell, M. M. Brister, M. Pittelkow, T. I. Solling and C. E. Crespo-Hernández, J. Am. Chem. Soc., 2018, 140, 11214-11218.

22 J. Pirillo, B. C. De Simone and N. Russo, Theor. Chem. Acc., 2016, 135, 8.

23 J. Pirillo, G. Mazzone, N. Russo and L. Bertini, J. Chem. Inf. Model., 2017, 57, 234-242.

24 S. Mai, A.-P. Wolf and L. González, J. Chem. Theory Comput., 2019, 15, 3730-3742.

25 Y.-G. Fang, Q. Peng, Q. Fang, W. Fang and G. Cui, ACS Omega, 2019, 4, 9769-9777.

26 Q. Peng, Y.-H. Zhu, T.-S. Zhang, X.-Y. Liu, W.-H. Fang and G. Cui, Phys. Chem. Chem. Phys., 2020, 22, 12120-12128.

27 M. C. DeRosa and R. J. Crutchley, Coord. Chem. Rev., 2002, 233-234, 351-371.

28 A. Sharonov, T. Gustavsson, S. Marguet and D. Markovitsi, Photochem. Photobiol. Sci., 2003, 2, 362-364.

29 R. J. Malone, A. M. Miller and B. Kohler, Photochem. Photobiol., 2003, 77, 158-164.

30 T. Gustavsson, A. Bányász, E. Lazzarotto, D. Markovitsi, G. Scalmani, M. J. Frisch, V. Barone and R. Improta, J. Am. Chem. Soc., 2006, 128, 607-619.

31 S. Yamazaki and T. Taketsugu, J. Phys. Chem. A, 2012, 116, 491-503.

32 J. Finley, P.-Å. Malmqvist, B. O. Roos and L. Serrano-Andrés, Chem. Phys. Lett., 1998, 288, 299-306.

33 T. H. Dunning, J. Chem. Phys., 1989, 90, 1007-1023.

34 P.-O. Widmark, P.-Å. Malmqvist and B. O. Roos, Theor. Chim. Acta, 1990, 77, 291-306.

35 M. Douglas and N. M. Kroll, Ann. Phys., 1974, 82, 89-155.

36 B. A. Hess, Phys. Rev. A: At., Mol., Opt. Phys., 1986, 33, 3742-3748.

37 M. Reiher, Wiley Interdiscip. Rev.: Dev. Biol., 2012, 2, 139-149.

38 F. Aquilante, R. Lindh and T. Bondo Pedersen, J. Chem. Phys., 2007, 127, 114107.

39 B. O. Roos, The Complete Active Space Self-Consistent Field Method and its Applications in Electronic Structure Calculations, John Wiley \& Sons, Ltd, 2007, pp. 399-445.

40 I. F. Galván, M. Vacher, A. Alavi, C. Angeli, F. Aquilante, J. Autschbach, J. J. Bao, S. I. Bokarev, N. A. Bogdanov and
R. K. Carlson, et al., J. Chem. Theory Comput., 2019, 15, 5925-5964.

41 N. Forsberg and P.-Å. Malmqvist, Chem. Phys. Lett., 1997, 274, 196-204.

42 J. P. Zobel, J. J. Nogueira and L. González, Chem. Sci., 2017, 8, 1482-1499.

43 G. Ghigo, B. O. Roos and P.-Å. Malmqvist, Chem. Phys. Lett., 2004, 396, 142-149.

44 B. O. Roos and P.-Å. Malmqvist, Adv. Quantum Chem., 2004, 47, 37-49.

45 L. De Vico, M. Olivucci and R. Lindh, J. Chem. Theory Comput., 2005, 1, 1029-1037.

46 M. A. Manae and A. Hazra, J. Phys. Chem., 2017, 121, 8147-8153.

47 H. G. Mautner, J. Am. Chem. Soc., 1956, 78, 5292-5294.

48 G. Cui and W.-H. Fang, J. Chem. Phys., 2013, 138, 044315.

49 D. Cremer and J. A. Pople, J. Am. Chem. Soc., 1975, 97, 1354-1358.

50 J. Boeyens, J. Cryst. Mol. Struct., 1978, 8, 317.

51 S. Mai, M. Richter, M. Heindl, M. F. S. J. Menger, A. Atkins, M. Ruckenbauer, F. Plasser, L. M. Ibele, S. Kropf and M. Oppel, et al., SHARC2.1: Surface Hopping Including Arbitrary Couplings-Program Package for Non-Adiabatic Dynamics, sharc-md.org, 2019.

52 D. R. Lide, CRC handbook of chemistry and physics, CRC press, 2004, vol. 85.

53 S. Mai, P. Marquetand and L. González, J. Phys. Chem., 2015, 119, 9524-9533.

54 J. A. Sánchez-Rodríguez, A. Mohamadzade, S. Mai, B. Ashwood, M. Pollum, P. Marquetand, L. González, C. E. Crespo-Hernández and S. Ullrich, Phys. Chem. Chem. Phys., 2017, 19, 19756-19766.

55 S. Bai and M. Barbatti, Phys. Chem. Chem. Phys., 2017, 19, 12674-12682.

56 L. Martnez-Fernández, I. Corral, G. Granucci and M. Persico, Chem. Sci., 2014, 5, 1336-1347.

57 Y.-G. Fang, D. Valverde, S. Mai, S. Canuto, A. C. Borin, G. Cui and L. González, J. Phys. Chem. B, 2021, 125, 1778-1789.

58 L. Serrano-Andrés, M. P. Fülscher and G. Karlström, Int. J. Quantum Chem., 1997, 65, 167-181.

59 S. Miertus, E. Scrocco and J. Tomasi, Chem. Phys., 1981, 55, 117-129. 